\title{
EDITORIAL
}

\section{The heart of a woman: addressing the gender gap in cardiovascular disease}

W omen's cardiovascular health has been a prominent theme in the medical news during the early months of 2011. In February, the American Heart Association (AHA) published updated effectiveness-based guidelines for the prevention of cardiovascular disease (CVD) in women (Mosca, L. et al. Circulation doi:10.1161/ CIR.0b013e31820faaf8), the first revision of these recommendations since 2007. One of the major changes in these new guidelines is the lowering of the threshold for the definition of 'high risk' of cardiovascular death, from $\geq 20 \%$ over 10 years to $\geq 10 \%$ over 10 years. The writing committee also called for the reporting of results from future cardiovascular clinical trials to be sex-specific. This goal is strengthened by the Heart disease Education, Research and Analysis, and Treatment (HEART) for Women Act that was reintroduced into the US Senate on 2 March. If the bill is passed, the sex-based reporting of medical data would become mandatory and an annual report to Congress on the quality of and access to care for women with CVD would be required. On the opposite side of the Atlantic, the European Society of Cardiology has also taken steps to bring CVD in women into the spotlight. Timed to coincide with International Womens' Day on 8 March, the society issued a statement containing a "red alert" for women's hearts (http://www.escardio.org/about/ press/press-releases/pr-11/Pages/International-WomenDay-2011.aspx?hit=DontMiss), and has published a special issue of the European Heart Journal focusing on the cardiovascular issues facing women. These stories raise several questions; what is the biological basis for a difference in cardiovascular disease risk and outcomes between the sexes? Why is cardiovascular health in women such a topical and important subject? Finally, what other steps are being taken to address these disparities?

The physiological and behavioral factors underlying the difference in cardiovascular disease risk and outcomes between men and women are diverse. However, the biology of sexual dimorphism in CVD is not fully understood and is a frontier of research. What is known is that genes, hormones, and autonomic function all contribute to these differences. Importantly, women experience greater fluctuation in hormone levels throughout life than men. CVD risk can be affected by the changes associated with pregnancy and menopause, and the use of hormonal contraceptives and hormone replacement therapy. The response to therapy can also differ between men and women. For example, angiotensin-converting-enzyme inhibitors and low-dose aspirin seem to be less effective in women than

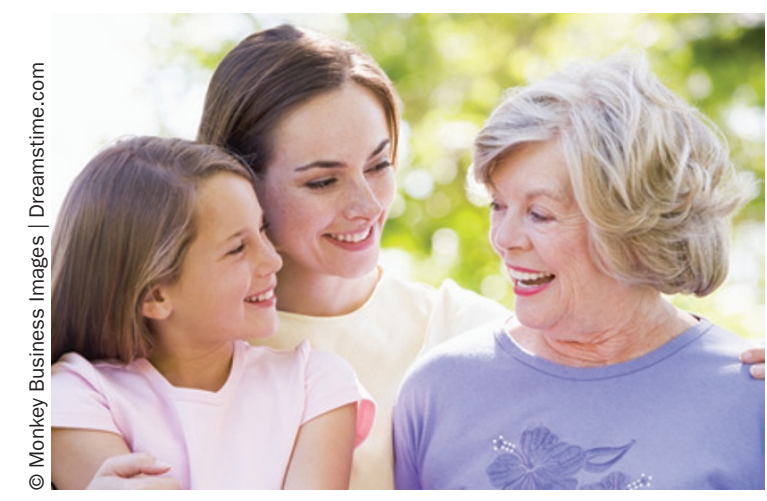

4 In the

USA, one

woman dies

from CVD

every minute

of every

day... 77 in men, and variation in response to statins between the sexes have also been reported (Arain, F. A. et al. Circ. J. 73, 1774-1782 [2009]). The differences in physiology between the sexes are, therefore, important to consider when assessing cardiovascular risk and treatment options.

CVD is the leading cause of mortality among women, accounting for $32 \%$ of deaths worldwide (5\% more than in men). By contrast, mortality from breast cancer is just $2 \%$ (http://www.who.int/healthinfo/global burden disease/ GBD_report_2004update_full.pdf). In the USA, one woman dies from CVD every minute of every day and, in 2007, almost half a million female lives were lost as a direct result of CVD. This figure was higher than that for deaths from cancer, respiratory disease, Alzheimer disease, and accidents combined. Worryingly, cardiovascular mortality among women aged 35-54 years actually seems to be increasing (Mosca, L. et al. Circulation doi:10.1161/CIR.0b013e31820faaf8).

Despite these disturbing statistics, the scale of the problem remains underestimated, both by the medical community and by women themselves. In a study of awareness about cardiovascular risk in women, primarycare physicians, gynecologists/obstetricians, and cardiologists often 'down-graded' the risk attributed to female patients. Women classified as being at 'intermediaterisk' of a cardiovascular events, as determined by the Framingham risk score, were significantly more likely to be assigned to a lower risk category then men with an identical Framingham score. Moreover, only one in five physicians knew that CVD caused more deaths in women than in men (Mosca, L. et al. Circulation 111, 499-510 [2005]). Women are generally diagnosed with CVD later in the disease process than men, because physicians might not immediately suspect cardiovascular etiology in
Alexandra King is the Senior Editor of Nature Reviews Cardiology.

Competing interests The author declares no competing interests. 
a woman, particularly if she is younger than $\sim 55$ years. Women are also less likely to receive medical therapies such as $\beta$-blockers, angiotensin-converting-enzyme inhibitors, and statins, and are less likely to be referred for cardiac catheterization than men. Thus, outcomes in women tend to be worse (Bugiardini, R et al. Eur. Heart J. doi:10.1093/eurheartj/ehr027). In addition, women are under-represented in cardiovascular clinical trials and so the 'evidence-based' therapies that they receive are often recommended on the basis that they are effective in men. The publication of the revised AHA guidelines and the work of several ongoing initiatives to promote awareness of female CVD will go a long way towards addressing these issues.

Disparities in outcomes are exacerbated by the lack of awareness about the magnitude of CVD risk among women themselves. A survey of women conducted in the USA revealed that only $42 \%$ of participants knew that CVD was the leading cause of death among their sex, and this figure was considerably lower among black, Hispanic, and younger ( $<45$ years) women than in older, white women (Mosca, L. et al. Circulation 109, 573-579 [2004]). In another study, only $56 \%$ of the women surveyed were able to describe the signs and symptoms of a heart attack and just 53\% said that they would call the emergency services if they thought that they were experiencing a cardiovascular event. The majority of women in this study were unaware of evidence-based preventative strategies; $69 \%$ cited multivitamins as a preventive measure, and 29\% thought aromatherapy was effective for the prevention of CVD (Mosca, L. et al. Circ. Cardiovasc. Qual. Outcomes 3, 120-127 [2010]). Although the mortality risk associated with breast and gynecological cancers is minimal when compared with CVD, women diligently attend screening sessions for these conditions, but do not seem to be as conscientious when it comes to the health of their hearts. Women often see themselves as 'care-givers' and are more likely to be concerned about the cardiovascular health of the men in their lives than they are about themselves. Pressure of work and family commitments often mean that women do not take time to visit their primary-care physician for a health check. Changing these behaviors and emphasizing that routine cardiovascular health checks, such as measurement of blood pressure and lipid levels, can be quick and simple is vital to improving cardiovascular outcomes among women.

Media coverage and fund-raising efforts for femalespecific cancers greatly exceed those for CVD. Raising the profile of female CVD is of the utmost importance and, to this end, several initiatives are underway. Now in its seventh year, the AHA's "Go Red for Women" campaign, symbolized by the 'red dress' motif, was designed to educate women that heart disease is their leading cause of death, and to inspire them to take action to protect their health. The initiative encourages women to learn the basic facts about heart disease in their sex, to share this information with their female friends and relatives, and to participate in 'red dress' events to raise awareness and funds for research. The Go Red for Women scheme also encompasses online tools that women can use to help them achieve hearthealthy lifestyles, including a heart health check-up and the "BetterU" nutrition and fitness program. The World Heart Federation is promoting the Go Red for Women initiative on a global level, through its relationships with national cardiovascular societies the world over.

"WomenHeart" is another organization in the USA that is dedicated to promoting women's heart health through advocacy, education and patient support. As part of the program, more than 500 women living with heart disease, so called "WomenHeart Champions", work to raises awareness about the importance of prevention and early detection, accurate diagnosis and optimum treatment of women's heart disease, and provide a support network for other women with cardiovascular conditions.

In Europe, the ESC have focused their efforts on highlighting to medical professionals the growing burden and under-appreciation of women's heart disease. Through the "Women at Heart" contest, national cardiovascular societies are encouraged to develop campaigns to raise awareness on women and CVD. The winning programs will receive funding from the ESC and will serve as a model for future campaigns in other countries.

Much has been achieved in the quest to raise awareness about CVD in women, to reduce disparities in cardiac care, and improve outcomes in women with heart disease, but there is still work to be done. CVD is a women's health issue; let us continue to treat it as such.

doi:10.1038/nrcardio.2011.49 